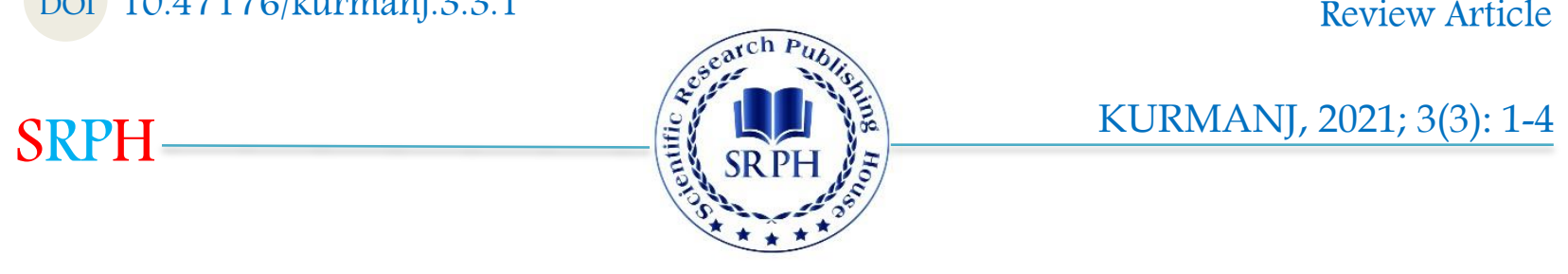

\title{
An Easy Way to Become a Polyglot
}

\author{
Mehrdad Jazayeri*
}

Faculty of Foreign Languages, Tehran

Central Branch, Islamic Azad

University, Tehran, Iran.

\section{*Corresponding Author: \\ \mansurjim95@gmail.com}

Received: 15 April, 2021

Accepted: 25 July, 2021

Published: 01 August, 2021

\begin{abstract}
Issues related to artificial languages and being an auxiliary language for a particular group of people to have some purposes which are related to politics, commerce, science, security, hidden actions, art and cinema or any other purposes. An artificial language can be constructed, planned, managed, developed and expanded by one person or a group of people. This study was conducted on the basis of the following observations: (1) to have a global language without a global culture. (2) to avoid having to choose between conflicting other languages. An artificial language is not a common subject of today's linguistics; in this study the major part is to create an artificial language to facilitate both written and spoken forms and to not forget every form of communication or being a logical language intended to express thoughts with clarity and you have to design it in a specific way and doesn't have to be very complicated. The present study aimed at communication between speakers of a certain group of closely related languages to learn it as fast as possible to open up international job opportunities, business opportunities and etc. Since a couple of decades ago, there has been a plethora of studies on official languages: an example of it would be the process of the language of Behasa Indonesia which is played a rule as a lingua Franca through the local Islands. Later, as Indonesian people achieved their union, they planned and programmed to change it into an official language, the language was developed in the aspects of grammar and vocabulary in a planning and constructing way. Results showed that why a language becomes global language has little to do with the number of people who speak it; A constructed language may not have the most speakers but given its global importance.
\end{abstract}

Keywords: Artificial language, Constructed language, Official language, Lingua Franca

\section{Introduction}

The idea that people should have a common language has long been an idea; most people in the world are bilinguals; This essay, much facilitates the acquirement of those who want to speak more than one language. Being a polyglot is one of those things that people want to accomplish one day, A good way to be a multilingual is to invent it.

A language is made up by a phonology, A morphology, A vocabulary and etc; It doesn't matter that you are the only one who speaks it.

Artificial languages are designed freely and little importance has been given to them.

\section{What is a Constructed Language?}

A constructed Language is a language whose phonology, grammar, and vocabulary are consciously devised and much easier to understand instead of having developed naturally.

Some languages like new French and Italian are naturaldeveloped languages and not purposefully constructed but they were purposefully expanded through many internal ethnic and local groups which were existed into these countries, the purpose was to become an unity for the cultural-linguistical aspect, To being bilingual or poly-lingual like such a country as Switzerland. 
In Iran, Persian and Azerbaijani Turkish have had the same situation, They have expanded and being adapted by many internal local ethnic groups, as historical reports stated, the language of people in special cities like Shiraz, Isfahan and other cities did not have this form of Persian which is named "Dari". Dari was the language of Novels and governors at the Sassanid era, and later adapted by public in some eastern Iranian lands and states such as Khorasan and then adapted by the rest of Iranian people who are now defined as Persian or Tajiki speakers (In Iran, Afghanistan and Tajikistan)

Such processes like that happened sometimes semiconsciously, like having a powerful literature and cultural backgrounds encouraged people to leave their own local languages and speak the main language of a community.

The most widely used lingua francas are defined as international auxiliary Languages, or Auxlan which are used in the entire world such as English, Spanish, Arabic and etc. all of these languages have both standard and local forms of speechs, sometimes these languages are expanded in the world not for a natural reason, but also for a purposed and planned strategy.

A conlang is not just expand or develop consciously, but it is also constructed in a conscious way. The optional parameters and human conscious choices are not including for programs of developing or expanding of a language, it is also including to construct lexical and grammatical structures of a language with the interference of conscious and the ability of human.

\section{Types of Conlangs}

Natural Conlang: this is used as a natural human language. It has it's own special scripts, forms of speeches, lexical and grammatical backgrounds; sometimes they have their specific vocabularies but many of them contain some loanwords which have been borrowed from the natural languages; the most famous example would be Esperanto, It has many loanwords while most of them come from the Latin and Slavic family of languages.

Scripture Sign Conlang: Like Computer Programming Conlang

Buddy Sign Conlang: a conlang may use buddy signs to emphasize the concepts, like a sign language used between people with deafness disability or also may used for spying and hidden actions.

Touch Conlang: which is used for touching scripts like Brail for people with blindness disability.

The two recent researches (buddy sign \& touch) are using whether as a special structure of a natural language for special people or purposes.

Some human made phenomena; It can be analyzed from a linguistic point of view such as music as an art like the sound of a bird or a bee which is reconstructed with a musical motif and by a musical instrument that imply certain real things.

\section{Suggestion}

We believe that we can construct a new auxiliary constructed language which is more simple, logical and practical. It can have both analytic and synthetic structures in an essential way. here are some recommendations:

\section{Logical base of the conlang based on a simple practical and meaningful alphabetic system}

The Latin script is the most widely used alphabetic writing system in the world; It is the basis for the largest number of alphabets of any writing system but to regulate and optimize it in a best way as we can, we are going to make some rules:

\subsection{Categorizing letters based on natural- philosophic phenomena or scientific subjects}

In Arabic, letters are categorized grammatically into two groups named solar and lunar. In Chinese, script morphemes are signed after a natural phenomena, like mountain.

we can Latinized this system and one of our best suggestions that we are going to discuss about is:

1) Categorizing the Latin Alphabets into four groups:

A. Letters based on the oldest philosophy: Wind, Water, Soil and Fire

B. Letters based on science: Gas, Liquid, Solid and Energetic

2) Using some shapes for the letters as a sign to emphasize or imply very fundamental concepts: for example choosing some directions:

$\mathrm{p}$ for right $\mathrm{q}$ for left $\mathrm{M}$ for up $\mathrm{W}$ for down

\subsection{Numerical System}

In Perso-Arabic alphabets, the letters in an special order named "Abjad Letters". this system has been used for some scientific actions like mathematics, music and also some strange sciences.

Some our researches discuss that the Abjad letters order is somehow very similar to Latin ordinary letters:

1) A B C D د I ( very similar in aspect of pronunciation. The letter $C$ sounds $P$ in some languages as Turkish language )

2) EFG j g० (partly similar)

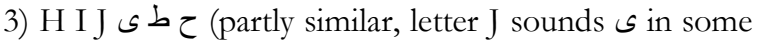
German and Danish. there isn't a relationship between I and $b$ at all )

4) KL M N S ل من (5) (exactly the same one )

5) O P w ع ف ص ن ص ص (No similarity found)

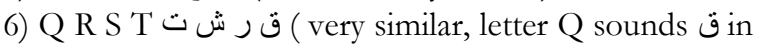


some languages and letter $S$ is somehow similar to ش)

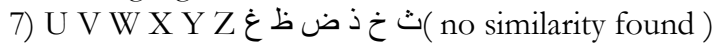

We are going to suggest you to Latinize this numerical system:

To create a mathematical function which is making a range of new words, like hashing functions on computer and network; hashing is an special function on computer science which is changing the words based on bits for the secure purposes.

As we know the 26 most widespread letters are the letters contained in the basic Latin alphabet; It could be concluded in other alphabets; below you'll find all 26 letters in some of other languages as well:

English: The English alphabet is a Latin alphabet consisting of 26 letters.

French: The French alphabet is based on the 26 letters of the Latin Alphabet.

German: The German alphabet consists of 26 basic letters like English.

Spanish: The Spanish alphabet is composed of 27 letters; it includes one extra letter $\tilde{n}$ that we don't have in English. Italian: The Italian alphabet only has 21 letters.

Turkish: The Turkish alphabet is a Latin-script alphabet; It consists of 29 letters.

The difdifficulty in creating an alphabet depends on how do you want to release it; the question is do you want to follow the same rules of actual languages or do you want to create a new pattern ? We did some patterns for it.

\{A B C D E F L N O P R T V \} It has only (13) letters a half of Latin scripts; These letters are all shared by the English alphabet; the pronunciation of a given constructed language word can largely be predicted from its spelling. In additional to these letters some languages use several accents that we can make a new pattern of them to diminish the number of alphabets.

1.1. $\ddot{A}$ is used on the A to make it sound like an English $\mathrm{I}: \ddot{\mathrm{A}}(\mathrm{ai})$

2. $\beta$ is used to make it sound like an English $Z: B$

1.2. $\ddot{E}$ is used on the $E$ to make it sound like an English

$\mathrm{I}: \mathrm{E}$ (i:)

3. $\mathrm{C}$ is used to make it sound like an English $\mathrm{K}$

4. $C$ is used under the $C$ to make it sound like an English S : Ç

5. $\mathrm{C}$ is used on the $\mathrm{C}$ to make it sound like an English $\mathrm{G}: \check{C}$

6. Doesn't need to use the letter H

7. Doesn't need to use the letter J

8. $\tilde{N}$ is used on the $\mathrm{N}$ to make it sound like an English $\mathrm{M}: \tilde{\mathrm{N}}$

9. $\ddot{O}$ is used on the $\mathrm{O}$ to make it sound like an English $\mathrm{U}: \ddot{O}$

10. Doesn't need to use the letter Q

11. Doesn't need to use the letter W
12. Doesn't need to use the letter $X$

13. Doesn't need to use the letter $Y$

\section{Mathematical and Geometrical Considerations}

We suggested you to construct a conlang based on mathematical or geometrical fundamentals, We emphasized some mathematical arguments in the above sentences but that was based on alphabets. Now we are going to use some mathematical and geometrical concepts to discuss this fact: How can we create a new language:

In public geometry we have three main linear matters and three main plates based on XYZ-Axis. we can use these materials to create words which are emphasizing the locations. As we said, $\mathrm{M}$ can use for up directions and we can use the word I for vertical line and the composition between these words, makes the word "Mi" which means up to a linear direction or A can use for a horizontal plate for example and the word "Ma" could mean over a flat.

These concepts and materials have the ability to be developed and we can use topology and topological concepts and implements to create words based on shapes and morphological considerations; in other words, the relation between structure and content will not be random and the use of it implies based on science.

\section{Grammatical Considerations}

The grammar is one of the most important part of a language, any ambiguity may destruct and disturb the language and the practices and applies of it.

The grammar that we are suggesting is based on inherent states and intrinsic verbs, which when you do an exact verb, it belongs to you and when your own are not going to do it but with the hand of someone else or things or situations, there should be a different grammatical case to describe it:

Doing yourself inherently

Doing yourself on another one's authority

Doing yourself with the cooperation of somebody's else or with a group of people Doing yourself with the force of an another one's verb, or decision.

All of the above sentences should have different grammatical buddies and transitive and intransitive verbs that can be made by using this theoretical method.

We want you to have both analytic and synthetic structures and use many different kinds of sentences. like nominal sentences, verbal sentences, and partial based like sentences based on adjectives, adverbs etc.. for example:

De sen nop Da'nen'ri (nominal sentence) (as the same in English) 
Sendo nop Da'nen'ri (verbal sentence) (as the same in Persian and Arabic) Da'nen'nop sendo (partial based sentence; based on complement)

All of the three above sentences can mean I go to school. De: I , sen: go , nop: to , Da'nen: School.

Sendo: as a verbal first part sentence, means I go. "do" is an ID stands for "De" meaning I. Note that they were just some examples.

\section{Conclusion}

This special issue brings together a set of paper that address the import of constructing a new language; our strategy is to develop this project in such a way that it can be naturally incorporated into the collection of real languages as an auxiliary language. It will put you in touch with people, specially if it has numerous native speakers, it begins to evolve and hence loses its constructed status.

A Constructed language in a natural way needs to add exceptions for every rule that we are going to create and also it needs to create a dictionary but in a free way it has no genders; It has very easy verb endings, adjectives remain the same for all words, there are no different endings to learn and there is no need to decide whether to use a polite form or an intimate form.

\section{References}

1. Presented Cleveland public library. A Companion to the Exhibit; Esperanto, Elvish and Beyond: The World of Constructed Languages. 2008.

2. Constructed Language by Wikipedia; The Free Encyclopedia

\section{KURMANJ}

Copyright: (C) 2021 The Author(s); This is an open-access article distributed under the terms of the Creative Commons Attribution License (http://creativecommons.org/licenses/by/4.0), which permits unrestricted use, distribution, and reproduction in any medium, provided the original work is properly cited.

Citation: Jazayeri M. An Easy Way to Become a Polyglot. KURMANJ, 2021; 3(3): 1-4.

https://doi.org/10.47176/kurmanj.3.3.1 